\title{
Inclusion relations for Bessel functions for domains bounded by conical domains
}

\author{
Chellakutti Ramachandran', Srinivasan Annamalai ${ }^{2}$ and Srikandan Sivasubramanian ${ }^{3 *}$
}

\author{
"Correspondence: \\ sivasaisastha@rediffmail.com \\ ${ }^{3}$ Department of Mathematics, \\ University College of Engineering, \\ Anna University, Tindivanam, \\ 604001, India \\ Full list of author information is \\ available at the end of the article
}

\begin{abstract}
In recent times, applications of Bessel differential equations have been effectively used in the theory of univalent functions. In this paper we study some subclasses of $k$-starlike functions, $k$-uniformly convex functions, and quasi-convex functions involving the Bessel function and derive their inclusion relationships. Further, certain integral preserving properties are also established with these classes. We remark here that $k$-starlike functions and $k$-uniformly convex functions are related to domains bounded by conical sections.

MSC: $30 C 45 ; 30 C 50$

Keywords: analytic function; univalent function; starlike function; convex function; subordination; $k$-starlike functions; $k$-uniformly convex functions; quasi-convex functions; Bessel function
\end{abstract}

\section{Introduction}

Let us consider the following second-order linear homogeneous differential equation (see for details [1] and [2]):

$$
z^{2} \omega^{\prime \prime}(z)+b z \omega^{\prime}(z)+\left[c z^{2}-u^{2}+(1-b) u\right] \omega(z)=0 \quad(u, b, c \in \mathbb{C})
$$

The function $\omega_{u, b, c}(z)$, which is called the generalized Bessel function of the first kind of order $u$, it is defined as a particular solution of (1.1). The function $\omega_{u, b, c}(z)$ has the familiar representation as

$$
\omega_{u, b, c}(z)=\sum_{n=0}^{\infty} \frac{(-c)^{n}}{n ! \Gamma\left(u+n+\frac{b+1}{2}\right)}\left(\frac{z}{2}\right)^{2 n+u} \quad(z \in \mathbb{C}) .
$$

Here $\Gamma$ stands for the Euler gamma function. The series (1.2) permits the study of Bessel, modified Bessel, and spherical Bessel function altogether. It is worth mentioning that, in particular:

(1) For $b=c=1$ in (1.2), we obtain the familiar Bessel function of the first kind of order $u$ defined by

$$
J_{u}(z)=\sum_{n=0}^{\infty} \frac{(-1)^{n}}{n ! \Gamma(u+n+1)}\left(\frac{z}{2}\right)^{2 n+u} \quad(z \in \mathbb{C})
$$

\section{Springer}

O2014 Ramachandran et al:; licensee Springer. This is an Open Access article distributed under the terms of the Creative Commons Attribution License (http://creativecommons.org/licenses/by/2.0), which permits unrestricted use, distribution, and reproduction in any medium, provided the original work is properly cited. 
(2) For $b=1$ and $c=-1$ in (1.2), we obtain the modified Bessel function of the first kind of order $u$ defined by

$$
I_{u}(z)=\sum_{n=0}^{\infty} \frac{1}{n ! \Gamma(u+n+1)}\left(\frac{z}{2}\right)^{2 n+u} \quad(z \in \mathbb{C}) .
$$

(3) For $b=2$ and $c=1$ in (1.2), the function $\omega_{u, b, c}(z)$ reduces to $\frac{\sqrt{2}}{\sqrt{\pi}} j_{u}(z)$ where $j_{u}$ is the spherical Bessel function of the first kind of order $u$, defined by

$$
j_{u}(z)=\frac{\sqrt{\pi}}{\sqrt{2}} \sum_{n=0}^{\infty} \frac{(-1)^{n}}{n ! \Gamma\left(u+n+\frac{3}{2}\right)}\left(\frac{z}{2}\right)^{2 n+u} \quad(z \in \mathbb{C})
$$

In [3], the author considered the function $\varphi_{u, b, c}(z)$ defined, in terms of the generalized Bessel function $\omega_{u, b, c}(z)$. From (1.2), it is clear that $\omega(0)=0$. Therefore, it follows from (1.2)

$$
\omega_{u, b, c}(z)=\left[2^{u} \Gamma\left(u+\frac{b+1}{2}\right)\right]^{-1} z^{u} \sum_{n=0}^{\infty} \frac{\left(\frac{-c}{4}\right)^{n}}{n ! \Gamma\left(u+n+\frac{b+1}{2}\right)} z^{2 n}, \quad \forall z \in \mathbb{C} .
$$

Let us set

$$
\varphi_{u, b, c}(z)=\sum_{n=0}^{\infty} b_{n} z^{n}
$$

where

$$
b_{n}=\frac{\left(\frac{-c}{4}\right)^{n}}{n ! \Gamma\left(u+n+\frac{b+1}{2}\right)} .
$$

Hence, (1.6) becomes

$$
\omega_{u, b, c}(z)=\left[2^{u} \Gamma\left(u+\frac{b+1}{2}\right)\right]^{-1} z^{u} \varphi_{u, b, c}\left(z^{2}\right)
$$

By using the well-known Pochhammer symbol (or the shifted factorial) $(\lambda)_{\mu}$ defined, for $\lambda, \mu \in \mathbb{C}$ and in terms of the Euler $\Gamma$ function, by

$$
\begin{aligned}
& \omega_{u, b, c}(z)=\left[2^{u} \Gamma\left(u+\frac{b+1}{2}\right)\right]^{-1} z^{u} \varphi_{u, b, c}\left(z^{2}\right), \\
& (\lambda)_{\mu}:=\frac{\Gamma(\lambda+\mu)}{\Gamma(\lambda)}= \begin{cases}1 & (\mu=0 ; \lambda \in \mathbb{C} \backslash\{0\}), \\
\lambda(\lambda+1) \cdots(\lambda+n-1) & (\mu=n \in \mathbb{N} ; \lambda \in \mathbb{C}),\end{cases}
\end{aligned}
$$

where it is being understood conventionally that $(0)_{0}=1$. Therefore, we obtain the following series representation for the function $\varphi_{u, b, c}(z)$ given by (1.6):

$$
\varphi_{u, b, c}(z)=z+\sum_{n=1}^{\infty} \frac{(-c)^{n} z^{n+1}}{4^{n}(\kappa)_{n} n !} \quad(z \in \mathbb{C}),
$$


where $\kappa=u+\frac{b+1}{2} \notin \mathbb{Z}_{0}^{-}, \mathbb{N}=\{1,2, \ldots\}$ and $\mathbb{Z}_{0}^{-}=\{0,-1,-2, \ldots\}$, and therefore

$$
\varphi_{u, b, c}(z)=z+\sum_{n=2}^{\infty} \frac{(-c / 4)^{n-1} z^{n}}{(\kappa)_{n-1}(n-1) !} \quad\left(\kappa:=u+\frac{b+1}{2} \notin \mathbb{Z}_{0}^{-}\right)
$$

where $\mathbb{N}:=\{1,2,3, \ldots\}$ and $\mathbb{Z}_{0}^{-}:=\{0,-1,-2, \ldots\}$. The function $\varphi_{u, b, c}$ is called the generalized and 'normalized' Bessel function of the first kind of order $u$. We note that by the ratio test, the radius of convergence of the series $\varphi_{u, b, c}(z)$ is infinity. Moreover, the function $\varphi_{u, b, c}$ is analytic in $\mathbb{C}$ and satisfies the differential equation $4 z^{2} \varphi^{\prime \prime}(z)+4 \kappa z \varphi^{\prime}(z)+c z \varphi(z)=0$. For convenience, we write $\varphi_{\kappa, c}(z)=\varphi_{u, b, c}(z)$. Let $\mathcal{A}$ denote the class of functions of the form

$$
f(z)=z+\sum_{n=2}^{\infty} a_{n} z^{n}
$$

which are analytic in the open unit disk $\mathbb{U}=\{z \in \mathbb{U}:|z|<1\}$. Let $\mathcal{S}$ be the subclass of $\mathcal{A}$ consisting of univalent functions in $\mathbb{U}$ with the normalized condition $f(0)=0=f^{\prime}(0)-1$. A function $f \in \mathcal{A}$ is said to be starlike of order $\eta$ if it satisfies $\Re\left(\frac{z f^{\prime}(z)}{f(z)}\right)>\eta(z \in \mathbb{U})$ for some $\eta$ $(0 \leq \eta<1)$ and we denote the class of functions which are starlike of order $\eta$ in $\mathbb{U}$ as $\mathcal{S}^{*}(\eta)$. Also, a function $f \in \mathcal{A}$ is said to be convex of order $\eta$ if it satisfies $\Re\left(1+\frac{z f^{\prime \prime}(z)}{f^{\prime}(z)}\right)>\eta(z \in \mathbb{U})$ for some $\eta(0 \leq \eta<1)$ and we denote by $\mathcal{C}(\eta)$ the class of all convex functions of order $\eta$ in $\mathbb{U}$. It follows by the Alexander relation that $f \in \mathcal{C}(\eta) \Leftrightarrow z f^{\prime} \in \mathcal{S}^{*}(\eta)$. The classes $\mathcal{S}^{*}(\eta)$ and $\mathcal{C}(\eta)$ were introduced by Robertson [4] (see also Srivastava and Owa [5]). Let $f \in \mathcal{A}$ and $g \in \mathcal{S}^{*}(\eta)$. Then $f$ is said to be close to convex of order $\gamma$ and type $\eta$ if and only if $\Re\left(\frac{z f^{\prime}(z)}{g(z)}\right)>$ $\gamma(z \in \mathbb{U})$ where $0 \leq \gamma<1$ and $0 \leq \eta<1$. The classes $\mathcal{K}(\gamma, \eta)$ were introduced by Libera [6] (see also Noor and Al-Kharsani [7], Silverman [8] and Shanmugam and Ramachandran [9]). Furthermore, we denote by $k-\mathcal{U C V}$ and $k-\mathcal{S T}(0 \leq k<\infty)$, two interesting subclasses of $\mathcal{S}$ consisting, respectively, of functions which are $k$-uniformly convex and $k$-starlike in $\mathbb{U}$ defined for $0 \leq k<\infty$ by

$$
k-\mathcal{U C V}:=\left\{f \in \mathcal{S}: \Re\left(1+\frac{z f^{\prime \prime}(z)}{f^{\prime}(z)}\right)>k\left|\frac{z f^{\prime \prime}(z)}{f^{\prime}(z)}\right|(z \in \mathbb{U})\right\}
$$

and

$$
k-\mathcal{S} \mathcal{T}:=\left\{f \in \mathcal{S}: \Re\left(\frac{z f^{\prime}(z)}{f(z)}\right)>k\left|\frac{z f^{\prime}(z)}{f(z)}-1\right|(z \in \mathbb{U})\right\} .
$$

The class $k-\mathcal{U C V}$ was introduced by Kanas and Wiśniowska in [10], where its geometric definition and connections with the conic domains were considered. The class $k-\mathcal{S T}$ was investigated in [11]. In fact, it is related to the class $k-\mathcal{U C V}$ by means of the wellknown Alexander equivalence between the usual classes of convex and starlike functions (see also the work of Kanas and Srivastava [12] for further developments involving each of the classes $k-\mathcal{U C V}$ and $k-\mathcal{S T}$ ). In particular, when $k=1$, we obtain $k-\mathcal{U C V} \equiv \mathcal{U C V}$ and $k-\mathcal{S T}=\mathcal{S P}$, where $\mathcal{U C V}$ and $\mathcal{S P}$ are the familiar classes of uniformly convex functions and parabolic starlike functions in $\mathbb{U}$, respectively. We remark here that the classes $k-\mathcal{U C V} \equiv \mathcal{U C V}$ and $k-\mathcal{S T}=\mathcal{S P}$ are related to the domain bounded by conical sections. Motivated by works of Kanas and Wiśniowska [10] and [11], Al-Kharsani and Al-Hajiry 
[13] introduced the classes $k$-uniformly convex functions and $k$-starlike functions of order $\eta(0 \leq \eta<1)$ as below:

$$
k-\mathcal{U C V}(\eta):=\left\{f \in \mathcal{S}: \Re\left(1+\frac{z f^{\prime \prime}(z)}{f^{\prime}(z)}-\eta\right)>k\left|\frac{z f^{\prime \prime}(z)}{f^{\prime}(z)}\right|(z \in \mathbb{U})\right\}
$$

and

$$
k-\mathcal{S T}(\eta):=\left\{f \in \mathcal{S}: \mathfrak{R}\left(\frac{z f^{\prime}(z)}{f(z)}-\eta\right)>k\left|\frac{z f^{\prime}(z)}{f(z)}-1\right|(z \in \mathbb{U})\right\}
$$

In the case when $k=0$ the inequalities (1.12) and (1.13) reduce to the well-known classes of starlike and convex functions of order $\eta$, respectively. Further, as mentioned earlier, for the special choices of $\eta=0$ and $k=1$ the class $k-\mathcal{U C V}(\eta)$ reduces to the class of uniformly convex functions introduced by Goodman [14] and the class $k-\mathcal{U C V}(\eta)$ reduces to the class of parabolic starlike functions studied extensively by Rønning [15] (see also the work of Ma and Minda [16]). If $f$ and $g$ are analytic in $\mathbb{U}$, then we say that the function $f$ is subordinate to $g$, if there exists a Schwarz function $w(z)$, analytic in $\mathbb{U}$ with $w(0)=0$ and $|w(z)|<1(z \in \mathbb{U})$, such that $f(z)=g(w(z))(z \in \mathbb{U})$. We denote this subordination by $f \prec g$ or $f(z) \prec g(z)(z \in \mathbb{U})$. In view of the earlier works studied by Kanas and Kanas et al. [10-12, 17-22], Sim et al. [23] and Al-Kharsani [24] defined the domain $\Omega_{k, \eta}$ for $0 \leq k<\infty$ as

$$
\Omega_{k, \eta}=\left\{u+i v:(u-\eta)^{2}>k^{2}(u-1)^{2}+k^{2} v^{2}\right\} .
$$

Note that, for $0<k<1$,

$$
\Omega_{k, \eta}=\left\{u+i v:\left(\frac{u+\frac{k^{2}-\eta}{1-k^{2}}}{k\left(\frac{1-\eta}{1-k^{2}}\right)}\right)^{2}-\left(\frac{v}{\frac{1-\eta}{\sqrt{1-k^{2}}}}\right)^{2}>1\right\}
$$

for $k>1$,

$$
\Omega_{k, \eta}=\left\{u+i v:\left(\frac{u+\frac{k^{2}-\eta}{k^{2}-1}}{k\left(\frac{1-\eta}{k^{2}-1}\right)}\right)^{2}+\left(\frac{v}{\frac{1-\eta}{\sqrt{k^{2}-1}}}\right)^{2}<1\right\} .
$$

The explicit form of the extremal function that maps $\mathbb{U}$ onto the conic domain $\Omega_{k, \eta}$ is given by

$$
Q_{k, \eta}(z)= \begin{cases}\frac{1+(1-2 \eta) z}{1-z} & k=0, \\ 1+\frac{2(1-\eta)}{\pi^{2}} \log ^{2}\left(\frac{1+\sqrt{z}}{1-\sqrt{z}}\right), & k=1, \\ 1+\frac{2(1-\eta)}{1-k^{2}} \sinh ^{2}(A(k) \operatorname{arctanh} \sqrt{z}), & 0<k<1, \\ \frac{(1-\eta)}{k^{2}-1} \sin ^{2}\left(\frac{\pi}{2 \kappa(t)} \mathcal{F}\left(\frac{\sqrt{z}}{\sqrt{t}}, t\right)\right)+\frac{k^{2}-\eta}{k^{2}-1}, & k>1,\end{cases}
$$

where $A(k)=\frac{2}{\pi} \arccos k, \mathcal{F}(\omega, t)$ is the Legendre elliptic integral of the first kind

$$
\mathcal{F}(\omega, t)=\int_{0}^{\omega} \frac{d x}{\sqrt{1-x^{2}} \sqrt{1-t^{2} x^{2}}}, \quad \kappa(t)=\mathcal{F}(1, t)
$$


and $t \in(0,1)$ is chosen such that $k=\cosh \frac{\pi \kappa^{\prime}(t)}{4 \kappa(t)}$. In view of the definition of subordination and the extremal function $Q_{k, \eta}(z)$,

$$
f \in k-\mathcal{S T}(\eta) \quad \Leftrightarrow \quad \frac{z f^{\prime}(z)}{f(z)} \prec Q_{k, \eta}(z)
$$

and

$$
f \in k-\mathcal{U C V}(\eta) \quad \Leftrightarrow \quad 1+\frac{z f^{\prime \prime}(z)}{f^{\prime}(z)} \prec Q_{k, \eta}(z)
$$

Therefore,

$$
\Re(p(z))>\Re\left(Q_{k, \eta}(z)\right)>\frac{k+\eta}{k+1} .
$$

Define $\mathcal{U C C}(k, \eta, \beta)$ as the family of functions $f \in \mathcal{A}$ such that

$$
\frac{z f^{\prime}(z)}{g(z)} \prec Q_{k, \eta}(z) \quad \text { for some } g(z) \in k-\mathcal{S T}(\beta)(0 \leq \beta<1)
$$

Similarly, we define $\mathcal{U} \mathcal{Q C}(k, \eta, \beta)$ as the family of functions $f \in \mathcal{A}$ such that

$$
\frac{\left(z f^{\prime}(z)\right)^{\prime}}{g^{\prime}(z)} \prec Q_{k, \eta}(z) \quad \text { for some } g(z) \in k-\mathcal{U C} \mathcal{V}(\beta)(0 \leq \beta<1) .
$$

We note that $\mathcal{U C C}(0, \eta, \beta)$ is the class of close to convex univalent functions of order $\eta$ and type $\beta$ and $\mathcal{U Q C}(0, \eta, \beta)$ is the class of quasi-convex univalent functions of order $\eta$ and type $\beta$. For $f \in \mathcal{A}$ given by (1.11) and $g(z)$ given by $g(z)=z+\sum_{n=1}^{\infty} b_{n+1} z^{n+1}$, the Hadamard product (or convolution) of $f(z)$ and $g(z)$ is given by

$$
(f * g)(z)=z+\sum_{n=1}^{\infty} a_{n+1} b_{n+1} z^{n+1}=(g * f)(z) \quad(z \in \mathbb{U})
$$

Note that $f * g \in \mathcal{A}$. For $\alpha_{j} \in \mathbb{C}(j=1,2, \ldots, q)$ and $\beta_{j} \in \mathbb{C} \backslash \mathbb{Z}_{0}^{-}(j=1,2, \ldots, s)$, the generalized hypergeometric function ${ }_{q} F_{s}\left(\alpha_{1}, \alpha_{2}, \ldots, \alpha_{q} ; \beta_{1}, \beta_{2}, \ldots, \beta_{s} ; z\right)$ is defined by the following infinite series (see the work of [25] and [26] for details):

$$
{ }_{q} F_{s}\left(\alpha_{1}, \alpha_{2}, \ldots, \alpha_{q} ; \beta_{1}, \beta_{2}, \ldots, \beta_{s} ; z\right)=\sum_{n=0}^{\infty} \frac{\left(\alpha_{1}\right)_{n} \cdots\left(\alpha_{q}\right)_{n} z^{n}}{\left(\beta_{1}\right)_{n} \cdots\left(\beta_{s}\right)_{n} n !}
$$

$\left(q \leq s+1 ; q, s \in \mathbb{N}_{0}=\mathbb{N} \cup\{0\}\right)$. Dziok and Srivastava [27] (also see [28]) considered the linear operator

$$
H\left(\alpha_{1}, \alpha_{2}, \ldots, \alpha_{q} ; \beta_{1}, \beta_{2}, \ldots, \beta_{s}\right): \mathcal{A} \rightarrow \mathcal{A}
$$

defined by the Hadamard product

$$
H\left(\alpha_{1}, \alpha_{2}, \ldots, \alpha_{q} ; \beta_{1}, \beta_{2}, \ldots, \beta_{s}\right) f(z)=z_{q} F_{s}\left(\alpha_{1}, \alpha_{2}, \ldots, \alpha_{q} ; \beta_{1}, \beta_{2}, \ldots, \beta_{s} ; z\right) * f(z)
$$


$\left(q \leq s+1 ; q, s \in \mathbb{N}_{0}=\mathbb{N} \cup\{0\}, z \in \mathbb{U}\right)$. If $f \in \mathcal{A}$ is given by (1.11), then we have

$$
H\left(\alpha_{1}, \alpha_{2}, \ldots, \alpha_{q} ; \beta_{1}, \beta_{2}, \ldots, \beta_{s}\right) f(z)=z+\sum_{n=1}^{\infty} \frac{\left(\alpha_{1}\right)_{n} \cdots\left(\alpha_{q}\right)_{n}}{\left(\beta_{1}\right)_{n} \cdots\left(\beta_{s}\right)_{n}} \frac{1}{n !} a_{n+1} z^{n+1} \quad(z \in \mathbb{U}) .
$$

Now, by using the above idea of Dziok and Srivastava [27], Deniz [2] introduced the $B_{\kappa}^{c}$ operator as follows:

$$
B_{\kappa}^{c} f(z)=\varphi_{\kappa, c} * f(z)=z+\sum_{n=1}^{\infty} \frac{(-c)^{n} a_{n+1} z^{n+1}}{4^{n}(\kappa)_{n} n !} .
$$

It easy to verify from the definition (1.20) that

$$
z\left[B_{\kappa+1}^{c} f(z)\right]^{\prime}=\kappa B_{\kappa}^{c} f(z)-(\kappa-1) B_{\kappa+1}^{c} f(z)
$$

where $\kappa=u+\frac{b+1}{2} \notin \mathbb{Z}_{0}^{-}$. In fact, the function $B_{\kappa}^{c}$ given by (1.20) is an elementary transformation of the generalized hypergeometric function. That is, it is easy to see that $B_{\kappa}^{c} f(z)=z_{0} F_{1}\left(\kappa ; \frac{-c}{4} z\right) * f(z)$ and also $\varphi_{\kappa, c}\left(\frac{-c}{4} z\right)=z_{0} F_{1}(\kappa ; z)$. In special cases of the $B_{\kappa}^{c}$-operator we obtain the following operators related to the Bessel function:

(1) Choosing $b=c=1$ in (1.20) or (1.21), we obtain the operator $\mathcal{J}_{u}: \mathcal{A} \rightarrow \mathcal{A}$ related with Bessel function, defined by

$$
\begin{aligned}
\mathcal{J}_{u} f(z) & =\varphi_{u, 1,1}(z) * f(z)=\left[2^{u} \Gamma(u+1) z^{\frac{1-u}{2}} J_{u}(\sqrt{z})\right] * f(z) \\
& =z+\sum_{n=1}^{\infty} \frac{(-1)^{n} a_{n+1} z^{n+1}}{4^{n}(u+1)_{n} n !}
\end{aligned}
$$

and its recursive relation

$$
z\left[\mathcal{J}_{u+1} f(z)\right]^{\prime}=(u+1) \mathcal{J}_{u} f(z)-u \mathcal{J}_{u+1} f(z)
$$

(2) Choosing $b=1$ and $c=-1$ in (1.20) or (1.21), we obtain the operator $\mathcal{I}_{u}: \mathcal{A} \rightarrow \mathcal{A}$ related with the modified Bessel function, defined by

$$
\begin{aligned}
\mathcal{I}_{u} f(z) & =\varphi_{u, 1,-1}(z) * f(z)=\left[2^{u} \Gamma(u+1) z^{\frac{1-u}{2}} I_{u}(\sqrt{z})\right] * f(z) \\
& =z+\sum_{n=1}^{\infty} \frac{a_{n+1} z^{n+1}}{4^{n}(u+1)_{n} n !}
\end{aligned}
$$

and its recursive relation

$$
z\left[\mathcal{I}_{u+1} f(z)\right]^{\prime}=(u+1) \mathcal{I}_{u} f(z)-u \mathcal{I}_{u+1} f(z) .
$$

(3) Choosing $b=2$ and $c=1$ in (1.20) or (1.21), we obtain the operator $\mathcal{S}_{u}: \mathcal{A} \rightarrow \mathcal{A}$ related with the spherical Bessel function, defined by

$$
\mathcal{S}_{u} f(z)=\left[\pi^{\frac{-1}{2}} 2^{\frac{u+1}{2}} \Gamma\left(\frac{u+3}{2}\right) z^{\frac{1-u}{2}} J_{u}(\sqrt{z})\right] * f(z)=z+\sum_{n=1}^{\infty} \frac{(-1)^{n} a_{n+1} z^{n+1}}{4^{n}\left(\frac{u+3}{2}\right)_{n} n !}
$$


and its recursive relation

$$
z\left[\mathcal{S}_{u+1} f(z)\right]^{\prime}=\left(\frac{u+3}{2}\right) \mathcal{S}_{u} f(z)-\left(\frac{u+1}{2}\right) \mathcal{S}_{u+1} f(z)
$$

Finally we recall the generalized Bernardi-Libera-Livingston integral operator, which is defined by

$$
L_{\gamma}(f)=L_{\gamma}(f(z))=\frac{\gamma+1}{z^{\gamma}} \int_{0}^{z} t^{\gamma-1} f(t) d t, \quad \gamma>-1 .
$$

\section{Preliminaries}

In proving our main results, we need the following lemmas.

Lemma 2.1 [29] Let $h$ be convex univalent in $\mathbb{U}$ with $h(0)=1$ and $\Re(v h(z)+\mu)>0(v, \mu \in$ $\mathbb{C})$. If $p$ is analytic in $\mathbb{U}$ with $p(0)=1$ then

$$
p(z)+\frac{z p^{\prime}(z)}{v p(z)+\mu} \prec h(z) \quad(z \in \mathbb{U}) \quad \Rightarrow \quad p(z) \prec h(z) \quad(z \in \mathbb{U}) .
$$

Lemma 2.2 [30] Let he convex in the open unit disk $\mathbb{U}$ and let $E \geq 0$. Suppose $B(z)(z \in \mathbb{U})$ is analytic in $\mathbb{U}$ with $\Re(B(z))>0$. If $g(z)$ is analytic in $\mathbb{U}$ and $h(0)=g(0)$. Then

$$
E z^{2} g^{\prime \prime}(z)+B(z) g(z) \prec h(z) \quad \Rightarrow \quad g(z) \prec h(z) .
$$

\section{Main results}

We study certain inclusion relationships for some subclasses of $k$-starlike functions, $k$ uniformly convex functions, and quasi-convex functions involving the Bessel equation. We reiterate that these classes of $k$-starlike functions and $k$-uniformly convex functions are related to domains bounded by conical sections.

Theorem 3.1 Let $c \geq 1$, and $h$ be convex univalent in $\mathbb{U}$ with $h(0)=1$ and $\Re(h(z))>0$. If a function $f \in \mathcal{A}$ satisfies the condition

$$
\frac{1}{1-\eta}\left[\frac{z\left(B_{\kappa}^{c} f(z)\right)^{\prime}}{B_{\kappa}^{c} f(z)}-\eta\right] \prec h(z) \quad(0 \leq \eta<1 ; z \in \mathbb{U})
$$

then

$$
\frac{1}{1-\eta}\left[\frac{z\left(B_{\kappa+1}^{c} f(z)\right)^{\prime}}{B_{\kappa+1}^{c} f(z)}-\eta\right] \prec h(z) \quad(0 \leq \eta<1 ; z \in \mathbb{U}) .
$$

Proof Let

$$
p(z)=\frac{1}{1-\eta}\left[\frac{z\left(B_{\kappa+1}^{c} f(z)\right)^{\prime}}{B_{\kappa+1}^{c} f(z)}-\eta\right] \quad(z \in \mathbb{U})
$$

where $p$ is an analytic function in $\mathbb{U}$ with $p(0)=1$. By using (1.21), we get

$$
(1-\eta) p(z)+\eta=\kappa \frac{z B_{\kappa}^{c} f(z)}{B_{\kappa+1}^{c} f(z)}-(\kappa-1)
$$


Differentiating logarithmically with respect to $z$ and multiplying by $z$, we obtain

$$
p(z)+\frac{z p^{\prime}(z)}{(1-\eta) p(z)+\eta+\kappa-1}=\frac{1}{1-\eta}\left[\frac{z B_{\kappa}^{c} f(z)}{B_{\kappa+1}^{c} f(z)}-\eta\right] .
$$

The proof of the theorem follows now by an application of Lemma 2.1.

Theorem 3.2 Let $f \in \mathcal{A}$. If $B_{k}^{c} f(z) \in k-\mathcal{S} \mathcal{T}(\eta)$, then $B_{\kappa+1}^{c} f(z) \in k-\mathcal{S} \mathcal{T}(\eta)$.

Proof Let

$$
s(z)=\frac{z\left(B_{\kappa+1}^{c} f(z)\right)^{\prime}}{B_{\kappa+1}^{c} f(z)} .
$$

From (1.21), we can write

$$
\kappa \frac{B_{\kappa}^{c} f(z)}{B_{\kappa+1}^{c} f(z)}=s(z)+\kappa-1
$$

Taking logarithmic differentiation and multiplying by $z$, we get

$$
\frac{z\left(B_{k}^{c} f(z)\right)^{\prime}}{B_{k}^{c} f(z)}=s(z)+\frac{z s^{\prime}(z)}{s(z)+\kappa-1} \prec Q_{k, \eta}(z) .
$$

Since $Q_{k, \eta}(z)$ is convex univalent in $\mathbb{U}$ and

$$
\Re\left(Q_{k, \eta}(z)\right)>\frac{k+\eta}{k+1},
$$

the proof of the theorem follows by Theorem 3.1 and condition (1.14).

Theorem 3.3 Let $f \in \mathcal{A}$. If $B_{k}^{c} f(z) \in k-\mathcal{U C V}(\eta)$, then $B_{\kappa+1}^{c} f(z) \in k-\mathcal{U C V}(\eta)$.

Proof By virtue of (1.12), (1.13), and Theorem 3.2, we obtain

$$
\begin{aligned}
B_{k}^{c} f(z) \in k-\mathcal{U C V}(\eta) & \Leftrightarrow z\left(B_{\kappa}^{c} f(z)\right)^{\prime} \in k-\mathcal{S} \mathcal{T}(\eta) \\
& \Leftrightarrow B_{\kappa}^{c} z f^{\prime}(z) \in k-\mathcal{S T}(\eta) \\
& \Rightarrow B_{\kappa+1}^{c} z f^{\prime}(z) \in k-\mathcal{S} \mathcal{T}(\eta) \\
& \Leftrightarrow B_{\kappa+1}^{c} f(z) \in k-\mathcal{U C V}(\eta)
\end{aligned}
$$

and hence the proof is complete.

Theorem 3.4 Let $f \in \mathcal{A}$. If $B_{k}^{c} f(z) \in \mathcal{U C C}(k, \eta, \beta)$, then $B_{\kappa+1}^{c} f(z) \in \mathcal{U C C}(k, \eta, \beta)$.

Proof Since

$$
\begin{aligned}
& B_{k}^{c} f(z) \in \mathcal{U C C}(k, \eta, \beta), \\
& \frac{z\left(B_{k}^{c} f(z)\right)^{\prime}}{k(z)} \prec Q_{k, \eta}(z) \quad \text { for some } k(z) \in k-\mathcal{S} \mathcal{T}(\beta) .
\end{aligned}
$$


For $g(z)$ such that $B_{\kappa}^{c} g(z)=k(z)$ we have

$$
\frac{z\left(B_{\kappa}^{c} f(z)\right)^{\prime}}{B_{\kappa}^{c} g(z)} \prec Q_{k, \eta}(z) .
$$

Letting

$$
h(z)=\frac{z\left(B_{\kappa+1}^{c} f(z)\right)^{\prime}}{B_{\kappa+1}^{c} g(z)} \quad \text { and } \quad H(z)=\frac{z\left(B_{\kappa+1}^{c} g(z)\right)^{\prime}}{B_{\kappa+1}^{c} g(z)} .
$$

We observe that $h(z)$ and $H(z)$ are analytic in $\mathbb{U}$ and $h(0)=H(0)=1$.

Now, by Theorem 3.2,

$$
B_{\kappa+1}^{c} g(z) \in k-\mathcal{S T}(\beta) \text { and } \Re(H(z))>\frac{k+\beta}{k+1} .
$$

Also note that

$$
z\left(B_{\kappa+1}^{c} f(z)\right)^{\prime}=\left(B_{\kappa+1}^{c} g(z)\right) h(z) .
$$

Differentiating both sides of (3.5), we obtain

$$
\frac{z\left(z\left(B_{\kappa+1}^{c} f(z)\right)^{\prime}\right)^{\prime}}{B_{\kappa+1}^{c} g(z)}=z \frac{\left(B_{\kappa+1}^{c} g(z)\right)^{\prime}}{B_{\kappa+1}^{c} g(z)} h(z)+z h^{\prime}(z)=H(z) \cdot h(z)+z h^{\prime}(z) .
$$

Now using the identity (1.21), we obtain

$$
\begin{aligned}
\frac{z\left(B_{\kappa}^{c} f(z)\right)^{\prime}}{B_{\kappa}^{c} g(z)} & =\frac{B_{\kappa}^{c}\left(z f^{\prime}(z)\right)}{B_{\kappa}^{c} g(z)} \\
& =\frac{z\left(B_{\kappa+1}^{c} z f^{\prime}(z)\right)^{\prime}+(\kappa-1) B_{\kappa+1}^{c}\left(z f^{\prime}(z)\right)}{z\left(B_{\kappa+1}^{c} g(z)\right)^{\prime}+(\kappa-1) B_{\kappa+1}^{c} g(z)} \\
& =\frac{\frac{z\left(B_{\kappa+1}^{c} z f^{\prime}(z)\right)^{\prime}}{B_{\kappa+1}^{c} g(z)}+(\kappa-1) \frac{B_{\kappa+1}^{c}\left(z f^{\prime}(z)\right)}{B_{\kappa+1}^{c} g(z)}}{\frac{z\left(B_{\kappa+1}^{c} g(z)\right)^{\prime}}{B_{\kappa+1}^{c} g(z)}+\kappa-1} \\
& =h(z)+\frac{z h^{\prime}(z)}{H(z)+\kappa-1} .
\end{aligned}
$$

From (3.4), (3.6), and the above equation, we conclude that

$$
h(z)+\frac{z h^{\prime}(z)}{H(z)+\kappa-1} \prec Q_{k, \eta}(z) .
$$

On letting $E=0$ and $B(z)=\frac{1}{H(z)+\kappa-1}$, we obtain

$$
\mathfrak{R}(B(z))=\frac{\mathfrak{R}(H(z)+\kappa-1)}{|H(z)+\kappa-1|^{2}}>0
$$

and the above inequality satisfies the conditions required by Lemma 2.2 . Hence

$$
h(z) \prec Q_{k, \eta}(z)
$$

and so the proof is complete. 
Using a similar argument to Theorem 3.4, we can prove the following theorem.

Theorem 3.5 Let $f \in \mathcal{A}$. If $B_{k}^{c} f(z) \in \mathcal{U} \mathcal{Q C}(k, \eta, \beta)$, then $B_{\kappa+1}^{c} f(z) \in \mathcal{U} \mathcal{Q C}(k, \eta, \beta)$.

Now we examine the closure properties of the integral operator $L_{\gamma}$.

Theorem 3.6 Let $\gamma>-\frac{k+\eta}{k+1}$. If $B_{\kappa}^{c} \in k-\mathcal{S T}(\eta)$ so is $L_{\gamma}\left(B_{\kappa}^{c}\right)$.

Proof From the definition of $L_{\gamma}(f)$ and the linearity of the operator $B_{\kappa}^{c}$ we have

$$
z\left(B_{\kappa}^{c} L_{\gamma}(f)\right)^{\prime}=(\gamma+1) B_{\kappa}^{c} f(z)-\gamma B_{\kappa}^{c} L_{\gamma}(f)
$$

Substituting $\frac{z\left(B_{\kappa}^{c} L_{\gamma}(f(z))\right)^{\prime}}{B_{\kappa}^{c} v L_{\gamma}(f(z))}=p(z)$ in (3.8) we may write

$$
p(z)=(\gamma+1) \frac{B_{\kappa}^{c} f(z)}{B_{\kappa}^{c} L_{\gamma}(f(z))}-\gamma
$$

On differentiating (3.9) we get

$$
\frac{z\left(B_{\kappa}^{c}(f(z))\right)^{\prime}}{B_{\kappa}^{c}(f(z))}=\frac{z\left(B_{\kappa}^{c} L_{\gamma} f(z)\right)^{\prime}}{B_{\kappa}^{c} L_{\gamma}(f(z))}+\frac{z p^{\prime}(z)}{p(z)+\gamma}=p(z)+\frac{z p^{\prime}(z)}{p(z)+\gamma} .
$$

By Lemma 2.1, we have $p(z) \prec Q(k, \eta)$, since $\Re(Q(k, \eta)+\gamma)>0$. This completes the proof of Theorem 3.6.

By a similar argument we can prove Theorem 3.7 as below.

Theorem 3.7 Let $\gamma>-\frac{k+\eta}{k+1}$. If $B_{\kappa}^{c} \in k-\mathcal{U C V}(\eta)$ so is $L_{\gamma}\left(B_{\kappa}^{c}\right)$.

Theorem 3.8 Let $\gamma>-\frac{k+\eta}{k+1}$. If $B_{\kappa}^{c} \in \mathcal{U C C}(k, \eta, \beta)$ so is $L_{\gamma}\left(B_{\kappa}^{c}\right)$.

Proof By definition, there exists a function

$$
K(z)=B_{\kappa}^{c} g(z) \in k-\mathcal{S T}(\eta)
$$

so that

$$
\frac{z\left(B_{\kappa}^{c}(f(z))\right)^{\prime}}{B_{\kappa}^{c}(g(z))} \prec Q_{k, \eta}(z) \quad(z \in \mathbb{U}) .
$$

Now from (3.8) we have

$$
\begin{aligned}
\frac{z\left(B_{\kappa}^{c} f\right)^{\prime}}{B_{\kappa}^{c}(g(z))} & =\frac{z\left(B_{\kappa}^{c} L_{\gamma}\left(z f^{\prime}\right)\right)^{\prime}+\gamma B_{\kappa}^{c} L_{\gamma}\left(z f^{\prime}(z)\right)}{z\left(B_{\kappa}^{c} L_{\gamma}(g(z))\right)^{\prime}+\gamma B_{\kappa}^{c} L_{\gamma}(g(z))} \\
& =\frac{\frac{z\left(B_{\kappa}^{c}\left(z f^{\prime}(z)\right)\right)^{\prime}}{B_{\kappa}^{c} L_{\gamma}(g(z))}+\frac{\gamma B_{\kappa}^{c}\left(z f^{\prime}(z)\right)}{B_{\kappa}^{c} L_{\gamma}(g(z))}}{\frac{z\left(B_{\kappa}^{c} c_{\gamma}(g(z))\right)^{\prime}}{B_{\kappa}^{c} L_{\gamma}(g(z))}+\gamma} .
\end{aligned}
$$


Since $B_{\kappa}^{c} g \in k-\mathcal{S} \mathcal{T}(\eta)$, by Theorem 3.6, we have $L_{\gamma}\left(B_{\kappa}^{c} g\right) \in k-\mathcal{S} \mathcal{T}(\eta)$. Taking $\frac{z\left(B_{\kappa}^{c} L_{\gamma}(g(z))\right)^{\prime}}{B_{\kappa}^{c} L_{\gamma}(g)}=$ $H(z)$, we note that $\Re(H(z))>\frac{k+\eta}{k+1}$. Now for $h(z)=\frac{z\left(B_{\kappa}^{c} L_{\gamma}(f(z))\right)^{\prime}}{B_{\kappa}^{c} L_{\gamma}(g(z))}$ we obtain

$$
z\left(B_{\kappa}^{c} L_{\gamma}(f(z))\right)^{\prime}=h(z) B_{\kappa}^{c} L_{\gamma}(g(z)) .
$$

Differentiating both sides of (3.12) yields

$$
\begin{aligned}
\frac{z\left(B_{\kappa}^{c}\left(z L_{\gamma}(f)\right)^{\prime}\right)^{\prime}}{B_{\kappa}^{c} L_{\gamma}(g)} & =z h^{\prime}(z)+h(z) \frac{z\left(B_{\kappa}^{c} L_{\gamma}(g)\right)^{\prime}}{B_{\kappa}^{c} L_{\gamma}(g)} \\
& =z h^{\prime}(z)+H(z) h(z) .
\end{aligned}
$$

Therefore from (3.11) and (3.13) we obtain

$$
\frac{z\left(B_{\kappa}^{c} f(z)\right)^{\prime}}{B_{\kappa}^{c} g}=\frac{z h^{\prime}(z)+H(z) h(z)+\gamma h(z)}{H(z)+\gamma} .
$$

This in conjunction with (3.10) leads to

$$
h(z)+\frac{z h^{\prime}(z)}{H(z)+\gamma} \prec Q(k, \eta)(z) .
$$

Let us take $B(z)=\frac{1}{H(z)+\gamma}$ in (3.15) and observe that $\Re(B(z))>0$ as $\gamma>-\frac{k+\eta}{k+1}$. Now for $A=0$ and $B$ as described we conclude the proof since the required conditions of Lemma 2.2 are satisfied.

A similar argument yields the following.

Theorem 3.9 Let $\gamma>-\frac{k+\eta}{k+1}$. If $B_{\kappa}^{c} \in \mathcal{U} \mathcal{Q C}(k, \eta, \beta)$ so is $L_{\gamma}\left(B_{\kappa}^{c}\right)$.

\section{Concluding remarks}

As observed earlier when $B_{\kappa}^{c}$ was defined, all the results discussed can easily be stated for the convolution operators $\mathcal{J}_{u} f(z), \mathcal{I}_{u} f(z)$, and $\mathcal{S}_{u} f(z)$, which are defined by (1.22), (1.23), and (1.24), respectively. However, we leave those results to the interested readers.

\section{Competing interests}

The authors declare that they have no competing interests.

\section{Authors' contributions}

All authors contributed equally to the writing of this paper. All authors read and approved the final manuscript.

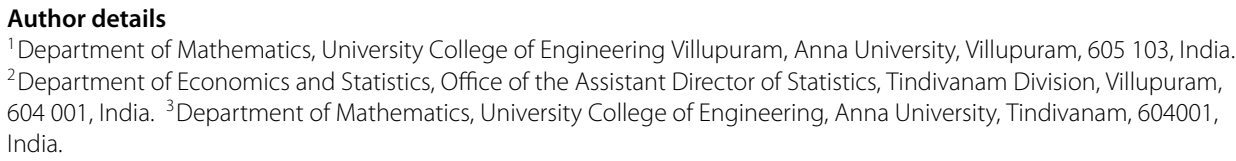

The authors sincerely thank the referee(s) for their valuable comments which essentially improved the manuscript. The work of the third author is supported by a grant from Department of Science and Technology, Government of India, vide ref: SR/FTP/MS-022/2012 under the fast track scheme. 


\section{References}

1. Baricz, Á: Generalized Bessel Functions of the First Kind. Lecture Notes in Mathematics. Springer, Berlin (2010)

2. Deniz, E: Differential subordination and superordination results for an operator associated with the generalized Bessel functions. Preprint

3. Deniz, E, Orhan, H, Srivastava, HM: Some sufficient conditions for univalence of certain families of integral operators involving generalized Bessel functions. Taiwan. J. Math. 15(2), 883-917 (2011)

4. Robertson, MS: On the theory of univalent functions. Ann. Math. (2) 37(2), 374-408 (1936)

5. Srivastava, HM, Owa, S (eds.) Current Topics in Analytic Function Theory. World Scientific, River Edge (1992)

6. Libera, RJ: Some radius of convexity problems. Duke Math. J. 31, 143-158 (1964)

7. Noor, Kl, Al-Kharsani, HA: Properties of close-to-convexity preserved by some integral operators. J. Math. Anal. Appl. 112(2), 509-516 (1985)

8. Silverman, $\mathrm{H}$ : On a class of close-to-convex functions. Proc. Am. Math. Soc. 36, 477-484 (1972)

9. Shanmugam, TN, Ramachandran, C: Komatu integral transforms of analytic functions subordinate to convex functions. Aust. J. Math. Anal. Appl. 4(1), 7 (2007)

10. Kanas, S, Wiśniowska, A: Conic regions and k-uniform convexity, II. Zeszyty Nauk. Politech. Rzeszowskiej Mat. 22, 65-78 (1998)

11. Kanas, S, Wiśniowska, A: Conic regions and k-uniform convexity. J. Comput. Appl. Math. 105(1-2), $327-336$ (1999)

12. Kanas, S, Srivastava, HM: Linear operators associated with $k$-uniform convex functions. Integral Transforms Spec. Funct. 9, 121-132 (2000)

13. Al-Kharsani, HA, Al-Hajiry, SS: Subordination results for the family of uniformly convex $p$-valent functions. J. Inequal. Pure Appl. Math. 7(1), 20 (2006)

14. Goodman, AW: On uniformly starlike functions. J. Math. Anal. Appl. 155(2), 364-370 (1991)

15. Rønning, F: A survey on uniformly convex and uniformly starlike functions. Ann. Univ. Mariae Curie-Skłodowska, Sect. A 47, 123-134 (1993)

16. Ma, WC, Minda, D: Uniformly convex functions. Ann. Pol. Math. 57(2), 165-175 (1992)

17. Kanas, S, Wiśniowska, A: Conic regions and k-starlike function. Rev. Roum. Math. Pures Appl. 45, $647-657$ (2000)

18. Kanas, S: Alternative characterization of the class k-UCV and related classes of univalent functions. Serdica Math. J. 25, 341-350 (1999)

19. Kanas, S: Techniques of the differential subordination for domains bounded by conic sections. Int. J. Math. Math. Sci. 38, 2389-2400 (2003)

20. Kanas, S: Differential subordination related to conic sections. J. Math. Anal. Appl. 317, 650-658 (2006)

21. Kanas, S: Subordination for domains bounded by conic sections. Bull. Belg. Math. Soc. Simon Stevin 15, 589-598 (2008)

22. Kanas, S: Norm of pre-Schwarzian derivative for the class of $k$-uniform convex and $k$-starlike functions. Appl. Math. Comput. 215, 2275-2282 (2009)

23. Sim, YJ, Kwon, OS, Cho, NE, Srivastava, HM: Some classes of analytic functions associated with conic regions. Taiwan. J. Math. 16(1), 387-408 (2012)

24. Al-Kharsani, HA: Multiplier transformations and k-uniformly P-valent starlike functions. Gen. Math. 17(1), 13-22 (2009)

25. Miller, SS, Mocanu, PT: Univalence of Gaussian and confluent hypergeometric functions. Proc. Am. Math. Soc. 110(2), 333-342 (1990)

26. Owa, S, Srivastava, HM: Univalent and starlike generalized hypergeometric functions. Can. J. Math. 39(5), 1057-1077 (1987)

27. Dziok, J, Srivastava, HM: Classes of analytic functions associated with the generalized hypergeometric function. Appl. Math. Comput. 103(1), 1-13 (1999)

28. Dziok, J, Srivastava, HM: Certain subclasses of analytic functions associated with the generalized hypergeometric function. Integral Transforms Spec. Funct. 14(1), 7-18 (2003)

29. Eeinigenburg, P, Miller, SS, Mocanu, PT, Reade, MD: General Inequalities, vol. 64, pp. 339-348. Birkhäuser, Basel (1983)

30. Miller, SS, Mocanu, PT: Differential subordinations and inequalities in the complex plane. J. Differ. Equ. 67, 199-211 (1978)

10.1186/1687-1847-2014-288

Cite this article as: Ramachandran et al.: Inclusion relations for Bessel functions for domains bounded by conical domains. Advances in Difference Equations 2014, 2014:288

\section{Submit your manuscript to a SpringerOpen ${ }^{\odot}$ journal and benefit from:}

- Convenient online submission

- Rigorous peer review

- Immediate publication on acceptance

Open access: articles freely available online

High visibility within the field

- Retaining the copyright to your article

Submit your next manuscript at $>$ springeropen.com 